\title{
British Association of Otolaryngologists Head and Neck Surgeons Summer Meeting, 7-8 September 2005, Edinburgh, Scotland, UK: otology abstracts
}

\section{A comparative study of bone-anchored hearing aid infection rates over a 2-year period: the effect of different wound dressings}

J Doshi, Y Karagama, D Buckley, I Johnson

From the Freeman Hospital, Newcastle Upon Tyne, UK

\section{Introduction}

Osseointegration and the avoidance of infection are crucial to successful implantation of a bone-anchored hearing aid (BAHA), but little evidence exists to suggest the best postoperative wound dressing to use. We compared the postoperative infection rate in patients receiving four types of BAHA wound dressings: ribbon gauze soaked in Tri-adcortyl (TAC) ointment; Mepitel (silicone); Allevyn (polyurethane); and Acticoat 7 (silver) with Allevyn.

\section{Method}

We conducted a prospective study of 160 consecutive BAHA patients treated at a tertiary referral centre between December 2002 and January 2005. Patients were reviewed at one and two weeks post-operatively at the nurse-led BAHA wound care clinic. Infection was defined as a positive wound swab culture or evidence of cellulitis at the BAHA site. The number of additional visits to the wound care clinic was noted.

\section{Results}

The BAHA post-operative infection rates were: 16 per cent (TAC), 50 per cent (Mepitel), 10 per cent (Allevyn) and 5 per cent (Acticoat 7 and Allevyn). The mean number of additional visits to the wound care clinic were: 1.5 (TAC), 3.7 (Mepitel), one (Allevyn) and 0.4 (Acticoat 7 and Allevyn).

\section{Conclusions}

A combination of Acticoat 7 and Allevyn dressings produced the lowest BAHA infection rate and the least number of additional visits to the wound care clinic. Our BAHA programme uses this combination as the dressing of choice.

\section{Survey of ossiculoplasty surgery in the UK}

H Khan, G Dhanasekar, N Malik, F Wilson, V Raut

From the Department of ENT, New Cross Hospital, Wolverhampton, UK

\section{Introduction}

The aim of this study was to assess and to quantify the current surgical practice of ossiculoplasty among British otolaryngology consultants, using a postal questionnaire.

\section{Method}

A questionnaire on ossiculoplasty surgery was sent to 539 UK consultant members of the British Association of Otolaryngologists Head and Neck Surgeons. The questionnaire comprised 31 questions covering: the consultant, the indication and timing of surgery (including types of prosthesis used), operative technique, and post-operative care. Replies were collected over a 12-week period; 280 replies were received.

\section{Results}

The response rate was 51.9 per cent. Of the 280 respondents, 179 (64.1 per cent) performed ossiculoplasty and 135 (48.2 per cent) performed revision ossiculoplasty. One hundred and fourteen (40.8 per cent) respondents used the patient's own ossicles (i.e. autografts), 58 (20.9 per cent) used cartilage, 61 (21.9 per cent) used cartilage and bone, 30 (10.9 per cent) used bone alone, and 155 (55.7 per cent) used an artificial prosthesis. One hundred and thirty-nine (49.7 per cent) respondents performed ossiculoplasty with primary tympanoplasty surgery. Ninety-one (32.8 per cent) respondents performed less than 10 ossiculoplasties per year, while 59 (21.3 per cent) respondents performed 15-20 ossiculoplasties per year. Seventy-five (26.8 per cent) respondents followed up their patients for one to three years, 37 (13.1 per cent) followed up their patients for five years, and 36 (12.9 per cent) followed up their patients for one year.

\section{Conclusions}

Our study attempted to quantify and to present a crosssectional survey of the current practice of ossiculoplasty surgery in the UK.

\section{Myringoplasty: does post-operative ear packing matter?}

V Nakhla, Y Takwoingi, A Sinha

From the City Hospital, Birmingham, UK

\section{Introduction}

Myringoplasty is a commonly performed otolaryngological procedure. A retrospective study was undertaken of 154 patients undergoing myringoplasty at the Birmingham City Hospital over a three-year period (January 2001 to January 2004). We specifically assessed whether postoperative ear dressings affected the outcome of the operation (i.e. intact tympanic membrane) and identified any other factors influencing outcome.

\section{Method}

Data were collected from patients' notes and analysed, including: age; site and size of perforation; grade of surgeon; surgical approach; use of post-operative ear dressings; complications; and audiometric outcome. The overall success rate of the operation (i.e. intact tympanic membrane at six months) was documented.

Results

The total number of myringoplasties over the three-year period was 170 and the data set was completed for 
154 patients. Patients' ages ranged from nine to 71 years (mean age, 34 years). Sixty-two per cent of the myringoplasties were performed by consultants and 38 per cent by trainees. The surgical approach was endaural in 82 per cent, and 89 per cent of procedures were closed with subcuticular sutures. The overall success rate of the operation was 80 per cent ( 85 per cent for consultants and 71 per cent for trainees). Audiometric improvement followed 74 per cent of the procedures. The mean follow-up period was seven months. The lowest success rate was found in procedures performed on subtotal perforations ( 75 per cent). The overall complication rate was 14 per cent; complications included infection and bismuth iodoform paraffin paste (BIPP) hypersensitivity. There was no significant difference in outcome between those patients who received a post-operative ear pack and those who did not $(p=0.29)$.

\section{Conclusions}

The authors conclude that the main factor influencing the success rate of myringoplasty was the size of the initial perforation. We recommend that no post-operative pack be applied following myringoplasty; this will save the patient the inconvenience of an out-patient visit and also the discomfort of pack removal.

\section{Local anaesthesia for Grommet insertion, EMLA vs.} Ametop-A comparative study

J R Nichani, S J Broomfield*, R V Nichani ${ }^{\dagger}$, S R Saeed ${ }^{\dagger}$, A E Camilleri*

From the Blackpool Victoria Hospital, Blackpool, the *South Manchester University Hospital, and ${ }^{\dagger}$ Manchester Royal Infirmary, Manchester, UK

\section{Introduction}

We aimed to assess the efficacy of EMLA as compared with Ametop in providing local anaesthesia for grommet insertion.

\section{Method}

A double blind, randomized, controlled trial was carried out to assess the efficacy of EMLA as compared with Ametop. The south Manchester local research ethics committee provided ethics approval. Forty-one patients were recruited and randomized to two groups. Visual analogue scores were used to assess pain control and satisfaction level.

\section{Results}

Twenty one patients were recruited into the EMLA group and 20 into the Ametop group. Data were analysed using Mann-Whittney-u test. The $p$-value for pain scores was 0.185 . The $p$-value for satisfaction score was 0.111 .

\section{Conclusions}

The difference in pain scores and satisfaction levels between EMLA and Ametop was not statistically significant. Traditionally, EMLA has been used as a topical local anaesthetic agent to provide pain control during grommet insertion. This study showed Ametop to be equally effective in providing local anaesthesia during this procedure. Ideally, EMLA requires approximately 60 minutes' application to provide adequate anaesthesia, whereas Ametop is effective in approximately 30 to 40 minutes. Both EMLA and Ametop are safe and effective local anaesthetics for grommet insertion.

\section{Patient information on the internet: are our websites on glue ear readable?}

\section{D Pothier}

From the Department of ENT, Gloucestershire Royal Hospital, Gloucester, UK

\section{Introduction}

I aimed to determine the readability of commonly accessed websites containing patient information on otitis media with effusion.

\section{Method}

The internet search engine Google was used to search web pages on UK servers. The search term 'glue ear' was used and the first 20 consecutive websites that contained patient information pages were analysed. The Flesch reading ease score and the Flesch-Kincaid reading grade level were used to assess the readability of the website. There was a strong negative correlation between the two different scoring systems $($ Pearson $=-0.93, p<0.001)$, which supported the validity of these results.

\section{Results}

The reading ages scores of the 20 sites ranged from 11.1 to 16.5 years, with a mean of 13.57 years (standard deviation, 1.55). Forty-five per cent of the Flesch reading ease scores were in the 'difficult' or 'fairly difficult' category, with 55 per cent falling into the 'standard' or 'fairly easy' categories. No site fell into the 'easy' or 'very easy' categories. These levels were well above the estimated reading age of the general UK population.

\section{Conclusions}

The poor readability of most of the commonly encountered internet websites about glue ear may confuse and frustrate many patients. These limitations should be recognized by website authors during the construction of their patient information websites so that the final result will be aimed at an appropriate level.

\section{Grommet otorrhoea: a prospective study in children with otitis media with effusion}

G Ramalingam, R Moorthy, A Scott

From the Royal Shrewsbury Hospital, Shrewsbury, UK

\section{Introduction}

We assessed the incidence of otorrhoea in paediatric patients undergoing grommet insertion for otitis media with effusion in our district general hospital department and compared it to rates in the published literature.

\section{Method}

A prospective, questionnaire-based study was conducted. Parents of children who met the inclusion criteria were invited to complete the questionnaire at the three-month follow-up visit. Operative findings and use of postoperative antibiotic drops were also recorded.

\section{Results}

Thirty-two children met the inclusion criteria (18 boys and 14 girls). All underwent bilateral grommet insertion (i.e. 64 ears were included in the study); five underwent adenoidectomy as well. Twenty-two ears received post-operative Sofradex eardrops. Seven out of the 64 ears (11 per cent) had purulent otorrhoea within four weeks following grommet insertion. Two ears in the antibiotic group (9 per cent) and five in the non-antibiotic group (12 per cent) had purulent otorrhoea within four weeks following grommet insertion. None of the five patients who underwent adenoidectomy developed otorrhoea.

\section{Conclusions}

The incidence of otorrhoea after grommet insertion was 11 per cent, in accordance with the 4-25 per cent rate 
published in the literature. Since the early 1990s, there have not been many published studies of grommet otorrhoea in the UK; hence, we reiterate the need for larger studies of grommet otorrhoea in the UK and of the current practice of grommet insertion in children.

\section{Masked mastoiditis in northern England}

S D Richards, V Mezentsev, G Back, D R Strachan

From the Bradford Royal Infirmary, Bradford, UK

\section{Introduction}

In the current, antibiotic, era, the presentation of acute mastoiditis may not be as described in ENT texts; it is often masked, with subtle clinical signs.

\section{Method}

We conducted an eight-year retrospective review of cases of acute mastoiditis treated at a teaching hospital. Patients with previous mastoid surgery were excluded. Using coding lists and theatre logs, 51 cases were identified. Seven case notes had been lost and two were incomplete.

\section{Results}

There was an equal number of adult and child patients. Seven patients were Asian, the remainder being white (35). Three cases presented with a fluctuant swelling and proceeded straight to surgery; all other cases (39) were initially treated conservatively. Of these 39 , eight settled with conservative management and 31 required surgery. Of the 31 requiring surgery after initial conservative management, 19 (45 per cent) had masked mastoiditis (i.e. none had a postauricular swelling, fluctuation or anterior displacement of the pinna), although six presented with complications. Surgery found cholesteatoma in 50 per cent and mucosal disease in 50 per cent.

\section{Conclusions}

Whilst the causative organisms and culture sensitivities were comparable with those in the published literature, the duration of symptoms was longer (20 days) and only 55 per cent of patients had classical signs of acute mastoiditis (i.e. less than the 56-100 per cent rate in other published studies). The use of antibiotics prior to admission was rare, but this finding may be spurious and due to poor documentation. In our patient population, mastoiditis often did not present in the classical fashion. We propose the need for clear guidelines for the referral of unresolved ear infections.

\section{Early results of titanium ossiculoplasty: a UK experience of the Kurz titanium prosthesis}

M Siddiq, V V Raut

From the New Cross Hospital, Wolverhampton, UK

\section{Introduction}

The aim of this study was to prospectively assess early results for titanium partial ossicular replacement prostheses (PORP) and total ossicular replacement prostheses (TORP) in the treatment of chronic ear disease.

\section{Method}

We analysed 33 consecutive patients treated at a tertiary referral centre between January 2004 and September 2004. Titanium ossiculoplasty was performed in isolation or as an accompaniment to surgery for chronic ear disease. Twenty-two PORPs and 11 TORPs were performed, together with primary or revision chronic ear surgery, by the senior author (VVR). Sliced cartilage was used as a standard to cover all prostheses. Follow up ranged from three to 12 months. Hearing results were assessed by comparing pre- and post-operative pure tone averages as well as closure of the air-bone gap (ABG). Extrusion rates were assessed.

Results

Ninety-one per cent of patients achieved an ABG of $<30 \mathrm{~dB}$ and 58 per cent achieved an $\mathrm{ABG}$ of $\angle 20 \mathrm{~dB}$, whereas 73 per cent achieved an ABG of $<20 \mathrm{~dB}$ using American Association of Otology-Head and Neck Surgery criteria $(0.5 / 1 / 2 / 3 \mathrm{kHz})$. The mean improvement in $\mathrm{ABG}$ at three months over the four frequency range $(0.5 / 1 / 2 / 4 \mathrm{kHz})$ was $7.6 \mathrm{~dB}$. The mean improvement in ABG over the $0.5 / 1 / 2 /$ $3 \mathrm{kHz}$ range was $8.4 \mathrm{~dB}$. The mean improvement in air conduction (AC) at three months over the $0.5 / 1 / 2 / 4 \mathrm{kHz}$ range was $10 \mathrm{~dB}$. The mean improvement in $\mathrm{AC}$ over the $0.5 / 1 / 2$ / $3 \mathrm{kHz}$ range was $11 \mathrm{~dB}$. Better results were found for PORPs than for TORPs. At the time of writing, no cases of extrusions or sensorial hearing loss had been seen.

\section{Conclusions}

The Kurz titanium prostheses showed good hearing results. They were easy to use and did not extrude when covered by cartilage. Long term results are awaited.

\section{Criteria used for magnetic resonance imaging screening of} suspected acoustic schwannoma: a retrospective study

S Somasheka, G Ramalingam, A Scott

From the Royal Shrewsbury Hospital, Shrewsbury, UK

\section{Introduction}

The criteria for magnetic resonance image (MRI) scanning in patients with suspected vestibular schwannoma were studied retrospectively in a group of patients and compared with published guidelines in the literature in order to identify avoidable referrals and thus the potential for reduction in waiting times and expenditure.

\section{Method}

Radiology department records in the Royal Shrewsbury Hospital were accessed and a random list assembled of 100 patients who had received MRI between June and December 2004. The records of 84 patients were found to be appropriate for study of the criteria. Patients' audiovestibular symptoms and audiometry results were analysed and compared with the widely accepted northern England and Yorkshire guidelines published in the literature.

\section{Results}

Seventy-one out of 84 patients ( 85 per cent) satisfied the criteria for MRI referral, while 15 per cent failed this criteria. Thirty-four out of 71 ( 48 per cent) patients had unilateral tinnitus, and 46 out of 71 ( 65 per cent) had asymmetric hearing loss of more than $20 \mathrm{~dB}$ in at least two adjacent frequencies. Twelve out of 71 (17 per cent) patients had Ménière's triad of symptoms. Two out of 71 (0.02 per cent) patients were found to satisfy the guidelines solely based on their duration of hearing loss, and one patient had facial palsy with asymmetry less than that $20 \mathrm{~dB}$ to fit the criteria. Eleven out of the 13 patients who failed the criteria had undergone an MRI scan for dizziness in order to rule out any central pathology, one patient had dizziness and slurred speech, and one patient had asymmetrical hearing loss less than that stipulated in the recommended guideline.

\section{Conclusions}

Eighty-five per cent of the patients studied satisfied the recommended criteria for MRI scanning. Fourteen per 
cent of the referrals that did not satisfy the recommendations received the MRI scan to rule out serious central nervous system pathology. One patient's MRI scan could have been avoided. In the period studied, two cases of acoustic neuroma and one case of temporal lobe lesion were discovered; all three of these patients satisfied the referral criteria.

\section{Influence of the European working time directive on post-surgical grommet follow up: a UK-based survey}

D H R Weerasinghe, W M Dudley, M Trotter, H R Hathurusinghe, N C Molony

From the ENT Department, Russells Hall Hospital, Dudley, West Midland, UK

\section{Introduction}

We aimed to ascertain the current practice of UK-based ENT consultants regarding follow up after grommet insertion. The study was prompted by time pressures on ENT clinics, from two conflicting areas. The European working time directive (EWTD) has resulted in a reduction of the number of hours worked by junior medical staff. However, this conflicts with current pressure to assess new patients within a set waiting time. Thus, a survey was undertaken to establish current practice and to investigate alternative strategies for grommet insertion follow up, in light of the EWTD.

Method

Consultant members of the British Association of Otorhinolaryngology Head and Neck Surgery in the
United Kingdom were sent an anonymous postcard questionnaire.

Results

Five hundred and twenty-one questionnaires were sent and 288 returned (55 per cent). Of these respondents, 279 (97 per cent) undertook grommet insertion. Of these surgeons, 240 (86 per cent) followed up their patients in the ENT clinic. Thirty-nine respondents did not pursue any patient follow up, three discharged their patients back to primary care, and the rest (36) had their patients seen by community or hospital audiologists or nurse specialists. One hundred and seventy-seven (74 per cent) respondents reviewed patients in their clinics and continued follow up until no complications were detected. One hundred and seventy-nine (64 per cent) respondents considered review by suitably trained staff in the community to be an acceptable alternative if available. Only 11 (4 per cent) respondents had reviewed their practice following implementation of the EWTD.

\section{Conclusions}

Follow up after grommets insertion is labour-intensive. However, very little change of practice has occurred since implementation of the EWTD. The majority of consultants would be willing to change their current practice should a suitable alternative be available. In the future, there will be increased pressure on clinics due to the EWTD and to the structured teaching programmes planned for junior doctors; this will result in fundamental changes in patient follow up. 